\section{Do nurses do it better?}

\section{S Rafferty, J S Elborn}

\section{Can nurse led clinics produce outcomes in respiratory care that are as effective as those produced by respiratory physicians?}

$\square$ he role of respiratory nurse specialists (RNS) in secondary care has increased dramatically during the past 10 years as a result of an increase in the skills and expertise of nurses and pressures to improve access of patients to appropriate care services.

The remit of the RNS is clinical and consultative-encompassing education, support, organisation of care, and the application of research. ${ }^{1}$ However, it is difficult to identify which patient outcomes are directly attributable to the actions of nurses. A recent systematic review in primary care concluded that nurse practitioners can provide high quality care for patients which is of similar quality to-or better than-that provided by general practitioners. ${ }^{2}$ In secondary care, however, it is less certain whether outpatient services can be provided by nurses. Studies in rheumatology and Parkinson's disease clinics show an equivalence between specialist nurse or nurse practitioner and specialist physician care. ${ }^{34}$ These studies, however, had methodological deficiencies such as small sample sizes, or there were referrals across the control and intervention groups to the nurse or physician.

In this issue of Thorax a randomised crossover trial by Sharples et al is published which explores the impact of a clinic for patients with stable bronchiectasis led by a nurse practitioner. The aims of the study were to determine whether a clinic led by a nurse practitioner can provide care that is as cost effective and safe as care provided by a respiratory physician. Eighty patients with stable bronchiectasis were randomised to receive care for two 1 year periods by either the nurse practitioner or a respiratory physician. A training programme was developed and there was regular medical supervision of the nurse practitioner. Patient care in the nurse practitioner group followed a medical management protocol. There were no significant differences in lung function and 12 minute walking test distance between nurse practitioner and respiratory physician care. The rate of hospital admissions for bronchiectasis per patient was slightly greater during nurse led care but, overall, this difference was not significant. Better health related quality of life ratings favoured nurse led care, but this was not statistically significant. This finding is not dissimilar to other studies which found that nurse led clinics resulted in equivalent health status ratings to care led by a doctor. ${ }^{67}$ The results of the study by Sharples et al support other research showing that nurses can provide care that is just as effective as doctors.

\section{". . nurses can provide care that is just as effective as doctors"}

There has been little research to evaluate the cost effectiveness of nurse led clinics in secondary care. A study of a Parkinson's disease nurse led clinic reported similar findings to those of Sharples et al, with the specialist nurse care being significantly more expensive than the specialist physician care. In primary care, nurse led clinics produced similar costs to physician care. ${ }^{7}$ The greater cost of the nurse practitioner led bronchiectasis care was primarily due to more admissions which lasted longer, and prescription of more intravenous antibiotics than with respiratory physician care. However, these were all judged to be appropriate by the respiratory physician. Interestingly, the costs of nurse practitioner led care decreased during the second year of the trial, while doctor costs rose. It is likely that experience gained over the study periods improved the nurses' depth of knowledge and expertise, allowing increased use of clinical judgement. A study of longer duration is required to determine whether the costs of nurse practitioner care would eventually equalise with those of respiratory physicians.

While not a measured phenomenon physicians in another study commented that they became more conscious of their own practice as they were forced to develop rigour and clarity to communicate with the nurse practitioners. ${ }^{8}$ This may account for some of the increase in the physician costs in the study by Sharples et al. ${ }^{5}$

This research is exciting as it is the first of its nature in respiratory care. It shows that nurses can work autonomously to produce outcomes in bronchiectasis which are as effective as those produced by respiratory physicians. This model may be helpful in providing care for chronic relapsing respiratory conditions such as bronchiectasis. Nurse led clinics could free up physician time to see new or clinically complex patients. This is especially important given the demands of government policy to reduce consultant waiting times to see new patients. ${ }^{9}$ Nurse led clinic care is acceptable to patients as measured from ratings of satisfaction with care which are equal to, and in some cases better than, physician care. ${ }^{36710-12}$ Process studies show that appropriately trained nurses perform assessments which are as thorough as physicians. ${ }^{8}$ However, further research is required to determine if the nurse led clinics are effective in other respiratory conditions such as chronic obstructive pulmonary disease or interstitial fibrosis.

Will nurse led clinics remove the nurse from the more traditional role of education, support, and organisation of care which patients also find valuable? This traditional role naturally lends itself to facilitating other initiatives such as the Department of Health's "The expert patient". ${ }^{13}$ However, research in rheumatology and Parkinson's disease suggests that nurses can effectively combine the medical role with the traditional nursing approach. ${ }^{3}$ The focus of nurse consultations is on nursing concerns of practical management of disability, explanations and education, and referral to other services, thereby resulting in a more holistic approach to care. This is very appropriate in patients with chronic disease requiring secondary care. Many patients will appreciate the continuity of care which an RNS can provide.

However, the increased time spent with patients adds to the costs of nurse led care. Combined physician/nurse or multidisciplinary clinics have proved to be effective in terms of clinical outcomes and health related quality of life in asthma. ${ }^{14}$ By contrast, the addition of a respiratory health worker to outpatient care improved mortality rates, but not health status. ${ }^{15}$ These studies are limited by flaws in methodology, such as choice of outcome measures or sample criteria. This is an area which requires further investigation. However, care is likely to be most safe and effective when working as part of a multidisciplinary team.

The level of nurse education, training, and experience is an important factor in the running of nurse led clinics. The advent of "supplementary prescribing" will allow specialist nurses to prescribe independently within a given framework when an initial diagnosis has been made by the physician. ${ }^{16}$ There may be concerns regarding the potential for missed 
diagnoses or prescribing errors in nurse led services. This did not appear to be a problem in the current study and has not been reported as a major issue in other complex disease clinics. ${ }^{34}$ In the United States a prerequisite to becoming a clinical nurse specialist is a masters degree. In the study by Sharples et al the nurse had a degree and a nurse practitioner qualification but nevertheless required further specialist education. An appropriate level of education, clinical expertise, and available support is important if the nurse is to exercise informed judgement in clinical decisions and prescribing. Professionally, the nurse must be confident that he/she is competent to fulfil expanded role requirements. ${ }^{17}$

The development of the RNS in the direct provision of care for patients with chronic respiratory disease is to be welcomed. The study by Sharples et al indicates that this is feasible and effective without compromising the quality of care. However, professionals do not work in isolation. It is important that long term care for chronic relapsing diseases is delivered by a team of professionals with the "expert patient" at the centre; such an approach is likely to maintain the patient's quality of life.

Thorax 2002;57:659-660

\section{Authors' affiliations}

S Rafferty, J S Elborn, Regional Respiratory Centre, Belfast City Hospital, Belfast BT9 7AB, UK

Correspondence to: Dr J S Elborn, Regional Respiratory Centre, Level 11, Belfast City Hospital, Belfast BT9 7AB, UK

stuart.elborn@bch.n-i.nhs.uk

\section{REFERENCES}

1 Wilson-Barnett J, Beech S. Evaluating the clinical nurse specialist. A review. Int J Nurs Stud 1994;31:561-71.

2 Horrocks S, Anderson E, Salisbury C. Systematic review of whether nurse practitioners working in primary care can provide equivalent care to doctors. BM 2002;324:819-23.

3 Hill J, Bird HA, Harmer R, et al. An evaluation of the effectiveness, safety and acceptability of a nurse practitioner in a rheumatology outpatient clinic. Br J Rheumatol 1994;33:283-8.

4 Reynolds H, Wilson-Barnett J, Richardson G Evaluation of the role of the Parkinson's disease nurse specialist. Int J Nurs Stud 2000;37:337-49

5 Sharples LD, Edmunds J, Bilton D, et al. A randomised controlled trial of nurse practitioner versus doctor led outpatient care in a bronchiectasis clinic. Thorax 2002;57:661-7.

6 Mundinger $M$, Kane R, Lenz E, et al. Primary care outcomes in patients treated by nurse practitioners or physicians. A randomised trial. JAMA 2000;283:59-68.

7 Venning P, Durie A, Roland $M$, et al. Randomised controlled trial comparing cost effectiveness of general practitioners and nurse practitioners in primary care. $B M$ 2000;320: 1048-53

8 Spitzer W, Sackett D, Sibley J, et al. The Burlington randomised trial of the nurse practitioner. N Engl J Med 1974;290:251-6.

9 Department of Health. Patient waiting time patient waiting lists; quality of patient care;
quality assurance; performance monitoring; quality assurance; performance monitoring; administro.

10 Kinnersley P, Anderson E, Parry K, et al. Randomised controlled trial of nurse practitioner versus of general practitioners care for patients requesting same day consultations in primary care. BM 2000;320: 1043-8.

11 Shum C, Humphreys A, Wheeler D, et al. Nurse management of patients with minor illnesses in general practice: multicentre, randomised controlled trial. $B M$ 2000;320: 1038-43

12 Hill J. Patient satisfaction in a nurse led rheumatology clinic. J Advan Nurs 1997;25:347-54.

13 Department of Health. The expert patient: a new approach to chronic disease management for the 21 st century. London: Stationery Office, 2001

14 Mayo P, Richman J, Harris H. Results of a programme to reduce admissions for adult asthma. Ann Intern Med 1990;1 12:864-71.

15 Littlejohns P, Baveystock CM, Parnell H, et al. Randomised controlled trial of the effectiveness of a respiratory health worker in reducing impairment, disability, and handicap due to chronic airflow limitation. Thorax 1991:46:559-64.

16 Department of Health. Patients to get quicker access to medicines. London: quicker access to medicines.
Department of Health, 2001.

17 United Kingdom Central Council. The scope of professional practice. London: United Kingdom Central Council for Nursing, Midwifery and Health Visiting, 1992. 